\title{
Le management face au judiciaire
}

\section{Un nouveau domaine d'enseignement et de recherche}

$\mathrm{L}$

e thème de ce numéro spécial est porté par les mutations qui affectent tant le droit que le management depuis la fin $\mathrm{du} \mathrm{XX}^{\mathrm{e}}$ siècle. Une transformation profonde de la relation entre ces deux disciplines oblige à repenser l'articulation des normes juridiques et des normes de management. Le besoin d'étudier les formes pratiques que prennent désormais la relation entre le droit et le management se manifeste tout particulièrement dans la multiplication, au sein des facultés de droit comme des écoles et facultés d'économie et de gestion, de programmes d'enseignement mêlant à parité ces deux disciplines.

Traditionnellement, l'articulation entre les deux systèmes normatifs est marquée par la subordination des normes et procédures managériales aux normes et procédures juridiques. En effet, la norme juridique s'inscrit historiquement dans un ordre hiérarchisé, au sein duquel la validité d'une norme inférieure dépend de sa conformité à une norme supérieure, avec au sommet de ce système 
pyramidal, les normes contenues dans la Constitution et la Déclaration des droits de l'homme et du citoyen (1789). Expression de la puissance étatique, l'ordre juridique s'est imposé comme le seul ordre légitime et souverain, dans lequel le respect des normes est garanti par une procédure et par une sanction appuyée sur la force publique. Cet « ordre juridique total » ramène à lui tous les autres. La promotion de l'ordre juridique s'est notamment manifestée par l'inclusion dans le programme d'enseignement de gestion d'un enseignement de droit dont le but était moins de produire des juristes que de valider l'adage selon lequel « $\mathrm{Nul}$ n'est censé ignorer la loi », en recourant, au besoin, à l'expertise d'un spécialiste. La conception traditionnelle de la légalité traçait ainsi les limites à l'intérieur desquelles le management pouvait développer librement ses logiques pragmatiques.

Cette situation s'est profondément transformée à partir de la deuxième moitié du $\mathrm{XX}^{\mathrm{e}}$ siècle sous l'effet de la mondialisation et des changements économiques, technologiques et politiques qu'elle véhicule. Face au marché mondial et dématérialisé, aux nouvelles technologies de l'information, l'État s'est retrouvé affaibli et concurrencé dans sa fonction normative par de multiples autorités, parmi lesquelles les entreprises transnationales. Agissant au niveau mondial, avec une puissance financière parfois proche de celle des États, elles se sont émancipées de la tutelle étatique mais aussi de son ordre juridique territorial et hiérarchisé. Dans ce contexte, la stabilité des normes requises pour la résolution des conflits vient se heurter au caractère éminemment dynamique du contexte des actions managériales et oblige à repenser la définition de la norme et sa place dans l'ordre social. En témoigne, le phénomène d'inflation législative, premier des symptômes d'une dégradation de la norme juridique adaptée aux réalités sociales qu'elle doit pourtant de moins en moins régir. Le phénomène va s'accentuer avec le développement, à côté du droit dit « dur » (hard law), d'un droit « souple » (soft law) qui, sans créer de véritable contrainte juridique, s'apparente par sa forme aux règles de droit classique ; plus récemment encore le développement de la responsabilité sociale de l'entreprise (RSE) marque l'intrusion et la confrontation au sein de l'entreprise des normes juridiques et des normes de management. La logique, et partant la suprématie de la norme juridique s'est ainsi trouvée fragilisée au point de compromettre la sécurité juridique : il est de plus en plus difficile pour le manager d'anticiper la conformité de ses actes à la norme juridique ; de là, une augmentation potentielle du nombre des procès et un allongement de la durée des procédures qui deviennent incompatibles avec une conduite efficace des affaires.

La crise normative peut se manifester, du point de vue du droit, par une confusion entre la logique juridique et la logique managériale dans l'entreprise, toutes deux ayant vocation à répondre à l'exigence de gestion interne et externe des risques liés à son activité. D'aucuns n'hésitent pas à parler ici d'une «managérialisation du droit » entendue comme une appropriation et transformation de la norme juridique par la rhétorique managériale. Cela pose inévitablement la question de la responsabilité et celle, plus précisément, de savoir comment le juge appréhende, dans sa fonction de juger, les choix et décisions managériaux, au regard notamment des programmes de conformité (à l'instar de celui exigé pour 
certaines entreprises par la loi n ${ }^{\circ} 2016-1691$ du 9 décembre 2016, dite loi Sapin II).

Cette situation de crise normative s'exprime, du point de vue du management, par la multiplication des contestations et une augmentation de l'ampleur des risques qui leurs son liés. La confusion des normes se manifeste autant lors de la recherche de responsabilité, dans la nécessité d'identifier l'auteur des actions, que dans la capacité de décrire les modalités précises de celles-ci et leurs conséquences.

Les articles du présent numéro spécial de la RFG illustrent la façon dont désormais, logique juridique et logique managériale se retrouvent à intervenir conjointement tant dans l'élaboration de l'action managériale que dans la résolution des conflits juridiques.

Cette situation d'internormativité peut résulter 1) de la mise en cause des limites qui organisent l'ordre juridique spatial et hiérarchisé et qui par ailleurs caractérisent la norme juridique, 2) de la nature de plus en plus complexe des normes et des processus de contrôle qu'exige leur mise en œuvre, au point d'aboutir à une confusion des normes, enfin, 3) des défis de l'innovation et des nouvelles technologies, qui exigent du droit et du management la production de mécanismes permettant d'assurer aussi bien la conformité des actions managériales à un système de normes préétablies que l'acceptabilité sociale de leurs conséquences.

\section{I - LA MISE EN CAUSE DES LIMITES}

La contribution de Manal El Bekkari et Catherine Mercier-Suissa sur « La gestion du risque de contrefaçon pour les PME dans le cadre de sous-traitance. Modalités et enjeux » montre comment méthodes formelles (juridiques) et informelles (managériales) doivent se combiner pour faire face, dans la gestion du risque de contrefaçon, à la diversité des règles juridiques et des contextes culturels nationaux. À ce premier niveau de complexité, s'ajoute celui lié aux caractéristiques propres des PME industrielles qui tiennent d'une part, à leur capacité financière qui limitent le recours à des procédures formelles couteuses, d'autre part, aux déficiences de leur organisation interne, sources de risques endogènes et exogènes.

De même, dans son article sur « Les manquements d'initiés en France. À qui profite le crime ?» dans lequel sont étudiées les décisions rendues par la Commission des sanctions de l'Autorité des marchés financiers (AMF) de 2001 à 2011, Elizabeth Fontany illustre comment le positionnement des acteurs, qu'ils soient professionnels de la finance ou membre des instances dirigeantes, par rapport aux limites de l'organisation, détermine aussi bien la nature même du délit que ses modalités et ses conséquences.

\section{II - LA CONFUSION DES NORMES}

Plusieurs contributions exposent comment le contrôle de l'application des normes juridiques sollicite simultanément, dans une sorte « d'internormativité droit et management » les normes juridiques et les normes de management ; cette internormativité peut tenir tant à la complexité même des organisations concernées, qu'à l'ambiguité intrinsèque des normes. 


\section{L'internormativité tenant à la complexité des organisations}

L'article de Oussama Ouriemmi et Benoît Gérard, «La responsabilisation de la Société Générale. Normes juridiques et management dans le volet civil du procès Kerviel », est une riche illustration d'un cas où le management «entre au prétoire ». L'affaire Société Générale/Kerviel est l'une des plus spectaculaires affaires de l'histoire financière, dans laquelle le trader d'une grande banque française, par sa seule fraude, a fait perdre presque 5 milliards d'euros à la banque. Or, comme le montrent les auteurs, cette affaire est aussi un terrain fertile de recherche interdisciplinaire où s'invitent, dans le même espace normes juridiques et normes de management. La décision judiciaire finale rendue, sur le montant des dommages-intérêts, le 23 décembre 2016 par la cour d'appel de Versailles (saisie comme cour de renvoi), laisse en effet à la charge du trader, pourtant reconnu pénalement responsable, à peine $0,02 \%$ du montant total du préjudice causé par sa fraude; le solde demeurant à la charge de la banque, par ailleurs condamnée dès 2008 par la Commission bancaire du fait de grave défaillances dans son contrôle interne. De ce point de vue, la décision se présente comme un véritable «cas de management » conduisant à la détermination d'un partage de responsabilités civiles. Avec l'article de Franck Aggeri et JeanMichel Saussois « La puissance des grandes entreprises mondialisées à l'épreuve du judiciaire. De l'affaire Volkswagen au dieselgate ", la complexité des processus impliqués prend une dimension supplémentaire. Cette affaire relative au contournement de normes environnementales ne met pas en cause les seuls membres de la société Volkswagen mais s'étend à tout un secteur et à la façon dont a pu se développer « une certaine gestion des illégalismes, faites de connivence et de relations d'accommodation entre le régulateur et le régulé ». Elle illustre l'importance de l'intervention de tiers extérieurs au couple régulateur-régulé (ONG, université, juges) qui a permis de révéler l'affaire; elle montre également l'effet de la diversité des systèmes juridiques auxquels sont confrontés les grandes entreprises mondialisées. Quant aux conséquences juridiques et managériales, elles concernent le management des entreprises, du fait de l'ampleur des amendes infligées et du retentissement des condamnations sur le marché du diesel ; elles concernent également la régulation des marchés par la façon dont elles éclairent les conditions requises pour un contrôle efficace des normes environnementales.

\section{Internormativité résultant de l'ambiguïté intrinsèque des normes}

C'est dans le domaine d'expertise de la norme comptable que l'article de Benjamin Di Gennaro, Véronique Blum et JeanMichel do Carmo Silva illustre un cas d'internormativité droit et management, découlant de l'ambiguïté propre de la norme comptable.Intitulé « Le règlement ANC 2014-05. Un cas d'internormativité comptable », l'article montre comment, dans le domaine des terrains de carrière et des redevances de foretage, l'adoption d'une nouvelle norme comptable par l'Autorité des normes comptables (ANC) est de nature à affecter directement le « business model » de l'entreprise. Préconisant une ventilation de la valeur extractible du gisement en stock 
et du tréfonds en immobilisation, le règlement ANC 2014-05 conduit en effet à la modification de la représentation de l'investissement à long terme ; ce faisant, et tout en visant l'homogénéisation du traitement comptable des coûts relatifs à l'acquisition des sites de carrières (pleine propriété et foretage), le règlement, qui instaure une seule norme pour deux chaînes de valeur différentes, affecte les stratégies managériales qui, en retour, affectent le sens et la portée de la norme elle-même.

Avec l'article de Gurvan Branellec et Stéphane Onnée sur «L'essor du crowdfunding immobilier. Entre logiques juridiques et logiques managériales », est en cause la façon dont l'irruption des nouvelles technologies de l'information et tout particulièrement celles qui résultent du développement d'Internet vient bouleverser les systèmes normatifs en place ainsi que les «business model» qui leur correspondaient. Le crowdfunding immobilier illustre en effet la façon dont Internet permet techniquement de contourner les intermédiaires financiers ainsi que les régulations qui les caractérisent et ce dans un domaine, l'immobilier, dont le bon fonctionnement est assuré par un cadre règlementaire complexe et rigoureux. Cela conduit à la nécessité de reconstruire un cadre normatif cohérent avec le nouvel écosystème généré par la technologie et à la mise en place de dispositifs managériaux permettant d'assoir leur «business model » dans la durée.

\section{III - LES DÉFIS DE L'INNOVATION ET DES NOUVELLES TECHNOLOGIES}

Les trois derniers articles proposés poussent plus avant le degré de complexité des relations entre droit et management. Chacun des articles montre en effet comment la nature même des faits managériaux met les normes au défi de la saisir soit du fait de leur vitesse d'exécution, du fait soit de leur complexité et de leur dispersion dans un monde globalisé, soit encore de leur indétermination dans un monde managérial où l'innovation, et plus encore l'innovation collective, est destinée à jouer un rôle de plus en plus important.

L'article de Marc Lenglet, « Coder décoder, recoder ? Ordres normatifs et enjeux de la régulation financière à l'ère du trading algorithmique », tend à démontrer que les nouvelles technologies de l'information, et précisément le trading algorithmique « à haute fréquence », sont à l'origine d'un basculement de l'ordre normatif juridique traditionnel vers un nouvel ordre normatif qui consacre la technologie et les organisations qui en découlent comme sources de régulation. «Mal compris, mal identifiés et difficiles à contrôler », les algorithmes, qui ne sont que des outils, viennent en quelque sorte « encapsuler » les pratiques de négociation renversant le rapport qui, jusque-là, prévalait entre le trader et son outil. De là l'idée forte que la technologie algorithmique «se fait régulation de par sa nature propre ». Depuis, l'actualité de l'intelligence artificielle et de la blockchain donne un fort écho à la démonstration.

Dans leur article «Responsabilité sociale des entreprises. Enjeux globaux et technologiques », David Restrepo Amariles, Arnaud Van Waeyenberge et Lorenzo Colombani observent une évolution semblable dans le domaine de la responsabilité sociale des entreprises (RSE). Par définition, la RSE dès son origine résulte d'une 
mise en cause des modes traditionnels de relation entre droit et management. Conçue initialement comme une dimension interne au management de l'entreprise liée à la reconnaissance de la présence d'externalités indésirables résultant de son activité, elle s'est, pour l'essentiel, développée à travers l'adoptions de codes de conduites multilatéraux ainsi que d'accords contractuels liés au droit du travail, au droit de l'environnement et à la protection des droits fondamentaux. Du point de vue procédural, ce domaine est le lieu d'innovations spectaculaires allant du développement de la « soft law » (« droit souple ») caractérisé par la prise en compte explicite des normes managériales par les juridictions, au développement, sous l'effet de l'essor des nouvelles technologies de dispositifs dits de « smart law », d'un régime automatique de sanction.

L'article de Mathias Béjean et Laurent Drai, «Innovation, collaboration et droit. L'internormativité dans les "labs" » montre comment l'évolution des processus d'innovation conduit à un nouveau degré de confusion entre le fait de l'action managériale et le droit supposé le régir. Cette mutation s'exprime aussi bien dans la généralisation de modes nouveaux d'organisation appelés "labs", « fab labs », ou « maker spaces », que dans les mutations du droit des brevets et des débats auxquels ils donnent lieu entre copyright et copyleft, entre open source et « creative commons ». Les nouvelles pratiques de l'innovation collective mettent au défi les normes juridiques, non du fait de la rapidité des phénomènes à réguler (i.e. trading haute fréquence), ni du fait de leur dispersion géographique et de leur complexité (i.e. la RSE) mais du fait de leur appartenance à l'espace indéterminé de l'imagination de réalités future. Et pourtant il devient de plus en plus nécessaire de penser et de répartir les droits de propriété relatifs à des objets dont la nature et le destin sont par nature inconnus des collectifs qui se donnent pour tâche de les élaborer. Le comble de la confusion des logiques juridiques et managériales est atteint quand on en vient à appliquer les méthodes d'innovation collective au droit lui-même, comme lorsque les méthodes de l' « open law » et du « law by design » s'expérimentent au sein du Legal Design Lab de Stanford.

À travers ces exemples il semble que la confrontation du management et du judiciaire soit destinée non seulement à faire partie de la vie quotidienne des managers et des juristes du fait de la subversion des limites qui donnaient au droit sa cohérence traditionnelle et au management son autonomie relative, mais encore qu'elle soit destinée à ouvrir de vastes champs de recherche aussi important pour la pratique que stimulant pour la théorie.

\section{RÉFÉRENCES BIBLIOGRAPHIQUES}

Baumard Ph. et Kobeissi N. (2015). « L'algorithme et l'ordre public », Archives de philosophie du droit, 58.

Benyekhlef K. (2015). Une possible histoire de la norme, Les normativités émergentes de la mondialisation, Thémis.

Capron M. et Quairel-Lanoizelée F. (2015). L'entreprise dans la société, Une question politique, La Découverte.

Carbonnier J. (2014). Sociologie juridique, PUF.

Cardon D. (2015). A quoi rêvent les algorithmes, nos vies à l'heure des big data, Seuil. 
Chevallier J. (2014). L'Etat post-moderne, droit et société, LGDJ.

Commaille J. (2015). A quoi nous sert le droit ?, Folio.

Conseil d'État (2013). Étude annuelle 2013, Le droit souple, La documentation française.

Frison-Roche M.-A. (2016). « L'arrêt Kerviel du 23 septembre 2016 : la compliance comme obligation de résultat pour tous et pour l'avenir », 19 octobre, MAFR.fr

Frydman B. et Jeuland E. (dir.) (2011). Le nouveau management de la justice et l'indépendance des juges, Thèmes et commentaires, Dalloz.

Laufer R. (2015). "Institution, droit et management : à propos de l'incertitude des limites », Mélanges Ch. Leben, Droit international et culture juridique, Pédone, p. 475-512.

Le Goff J. et Onnée S. (2017). Puissances de la norme, Défis juridiques et managériaux des systèmes normatifs contemporains, ems, Management et société. Martin-Chenut K. et de Quenaudon R. (dir.) (2016). La RSE saisie par le droit, Perspectives interne et internationale, A. Pedone éditeur.

Pezet E. et Sénéchal J. (dir.) (2014). Normes juridiques et normes managériales, Enjeux et méthode d'une nouvelle internormativité, Droit et société, $n^{\circ} 29$, LGDJ.

Ploquin X. (2016). «Un besoin de sincérité », Annales des Mines - Responsabilité et environnement, vol. 81, $\mathrm{n}^{\mathrm{o}} 1$, p. 72-75.

Supiot A. (2015). L'entreprise dans un monde sans frontières : perspectives économiques et juridiques, Dalloz.

Thibierge C. et al. (2014). La densification normative, Découverte d'un processus, Mare et Martin.

Thibierge C. et al. (2019). La force normative, Naissance d'un concept, LGDJ, Bruylant. 